\title{
Chemical profiling of complex biochemical mixtures from various seaweeds
}

\begin{abstract}
Yasuhiro Date $^{1,2}$, Kenji Sakata ${ }^{2}$ and Jun Kikuchi ${ }^{1,2,3,4}$
Development of an approach to describing and comparing complex biochemical mixtures presented by natural biomacromolecular sources is a promising new field in polymer science. The generation of a comprehensive strategy for evaluating and characterizing natural biomass mixtures presents a significant challenge. Here, we describe a chemical profiling methodology for seaweed biomass based on the comprehensive characterization of water-soluble, water-insoluble and solid-state components. An extraction method was developed for the nuclear magnetic resonance measurement of the water-insoluble seaweed components by modifying and optimizing physical pretreatments and extraction processes. Evaluating the compositional variations in various seaweeds from biomass profiles by principal component analysis score plots showed that the species clustered according to their taxonomic group differences. In addition, this profiling strategy revealed that it was possible to annotate the primary components that are characteristic of each taxonomic group, such as mannitol and laminaran in brown algae, D-galactose and agar in red algae, and xylopyranose and glucopyranose in green algae. Therefore, this profiling strategy was capable of discerning and evaluating individual characteristics among these seaweeds.
\end{abstract}

Polymer Journal (2012) 44, 888-894; doi:10.1038/pj.2012.105; published online 13 June 2012

Keywords: compositional profiling; multivariate statistical analysis; nuclear magnetic resonance (NMR); polysaccharide; seaweed biomass

\section{INTRODUCTION}

Polymer science generally involves uniform subunits that are polymerized from petroleum-derived building blocks, such as nylon and polyethylene, and does not usually focus on or produce polymers with complex chemical structures or mixtures with numerous and diverse macromolecular complexities. However, a vast majority of biomacromolecules in nature possess biochemically complex structures and thus present a significant challenge in the evaluation and characterization of the components of complexed biomacromolecules, such as land plant biomass and seaweed polysaccharides. Seaweed biomass, primarily composed of polysaccharides, has particularly low hydrophobic and crystalline polymers that make them distinct from cellulosic polymers that are widely put to practical uses. ${ }^{1,2}$ Therefore, a new field in polymer science will develop as the challenges in characterizing and understanding the biochemical complexities of various seaweed natural products are solved.

The ocean area surrounding Japan comprises the sixth largest exclusive economic zone in the world and possesses a great variety of marine resources, including fisheries and mineral resources. ${ }^{3}$ Of these resources, the biodiversity in marine ecosystems based on seaweeds in the coastal regions of Japan provides valuable biomass resources and has tremendous benefits to serve human needs. ${ }^{4}$ The biomass from seaweeds inhabiting the coastal regions are composed of various beneficial polysaccharides, such as alginic acid, fucoidan and agarose. ${ }^{1,5}$ Although a small portion of these beneficial polysaccharides have been used in both manufacturing and for pharmaceutical products, the vast majority remains unexploited for their potential industrial applications because of the paucity of evaluation and characterization techniques for the utility, applicability and availability of these natural products. Therefore, the development of a biopolymer analytical strategy designed to describe and assess the diverse characteristics of seaweed biomass is desirable.

The evaluation and characterization of compositional variations in seaweed biomass is important for developing a method to determine the biopolymer application and composition of seaweeds on the basis of their biomass characteristics. This evaluation and characterization is required to extract useful information from the seaweed biomass complexes obtained by comprehensively measuring these complicated biomacromolecules in untapped natural resources. From this viewpoint, a nuclear magnetic resonance (NMR)-based metabolomics approach is a powerful tool for comprehensively evaluating the metabolic profiles of biochemical complexes in natural ecosystems and has been used extensively to study metabolites from a wide range of biological systems in various environments. ${ }^{6-13}$ In addition, an NMR-based metabolomics approach has been applied to biomass

${ }^{1}$ RIKEN Plant Science Center, Yokohama, Kanagawa, Japan; ${ }^{2}$ Graduate School of Nanobioscience, Yokohama City University, Yokohama, Kanagawa, Japan; ${ }^{3}$ Graduate School of Bioagricultural Sciences, Nagoya University, Nagoya, Aichi, Japan and ${ }^{4}$ Biomass Engineering Program, RIKEN Research Cluster for Innovation, Yokohama, Kanagawa, Japan Correspondence: Professor J Kikuchi, RIKEN Plant Science Center, 1-7-22 Suehirocho, Tsurumi-ku, Yokohama, Kanagawa 230-0045, Japan.

E-mail: kikuchi@psc.riken.jp

Received 13 February 2012; revised 20 April 2012; accepted 27 April 2012; published online 13 June 2012 
profiling for land plant cell wall components. ${ }^{14,15}$ However, biomass profiling of water-soluble and water-insoluble (cell wall) components in diverse seaweeds has been largely unexplored in terms of using the comprehensive characterization afforded by an NMR-based metabolomics approach.

Here, the biomass profiling of biochemical complexes from a variety of seaweeds, including brown, green and red algae (16 species total), is described through the comprehensive characterization of water-soluble, water-insoluble and solid-state components. For the NMR analysis of water-insoluble components, an extraction process based on a DMSO- $\mathrm{d}_{6} /$ Pyridine $\mathrm{d}_{5}(4 / 1, \mathrm{v} / \mathrm{v})$ solvent system was modified and optimized. ${ }^{15}$ In addition, the compositional variations in and diversity of the water-soluble, water-insoluble and solid-state components were evaluated and compared by both NMR and attenuated total reflectance Fourier transform infrared (ATR-FTIR) spectroscopy, and subsequently subjected to multivariate statistical analysis.

\section{MATERIALS AND METHODS}

\section{Sample collection}

The seaweeds used in this study were collected on 11 August and 9 September 2010 from an intertidal area at Tenjin Island in Yokosuka City, Kanagawa, Japan (N35.222, E139.602). The collected samples were classified into brown, green and red algae (9, 4 and 3 species, respectively; Supplementary Table 1).

\section{NMR spectroscopy}

To evaluate the water-soluble components of seaweeds, lyophilized samples were crushed to a powder using an Automill machine (Tokken, Inc., Chiba, Japan) and suspended in a phosphate buffer solution $\left(0.1 \mathrm{M} \mathrm{K} \mathrm{K}_{2} \mathrm{HPO}_{4} / \mathrm{KH}_{2} \mathrm{PO}_{4}\right.$, $\mathrm{pH}$ 7.0) containing $90 \%$ deuterium oxide and $1 \mathrm{~mm}$ sodium 2,2-dimethyl-2silapentane-5-sulfonate (DSS) as an internal standard for NMR spectroscopy. One-dimensional (1D) Watergate (WG $)^{16}$ and Carr-Purcell-Meiboom-Gill (CPMG) spectra of these samples were acquired at $298 \mathrm{~K}$ on a $700 \mathrm{MHz}$ Bruker Biospin NMR instrument (AV700) equipped with an inverse (proton coils closest to the sample) gradient $5 \mathrm{~mm}$ Cryo ${ }^{1} \mathrm{H} /{ }^{13} \mathrm{C} /{ }^{15} \mathrm{~N}$ probe (Bruker Biospin, Rheinstetten, Germany). The WG pulse sequence is an effective technique for suppressing only the water signal, while retaining signals from both macromolecules and small molecules in the sample and is widely used in many complicated pulse sequences. ${ }^{16}$ On the other hand, CPMG spectra were also acquired to attenuate the broad signals from the macromolecules (for example, polysaccharide, protein and lipoproteins) and enhance sharper signals, such as those from small molecules. ${ }^{17-19}$ This enables the identification and relative quantification of small molecules in both the aliphatic and aromatic regions of the spectra. Therefore, WG and CPMG pulse sequences were used to characterize, compare and evaluate the macromolecules and small molecules in the water-soluble components. The two-dimensional (2D) ${ }^{1} \mathrm{H}-{ }^{13} \mathrm{C}$ heteronuclear single quantum coherence (HSQC) method for NMR measurements has been described previously. ${ }^{20-22}$ Briefly, a total of 128 complex f1 $\left({ }^{13} \mathrm{C}\right)$ and 2048 complex $\mathrm{f} 2\left({ }^{1} \mathrm{H}\right)$ points were recorded from 24 scans per $\mathrm{fl}$ increment. The obtained spectral widths were 150 and 14 p.p.m. for $\mathrm{f} 1$ and $\mathrm{f} 2$, respectively, and the NMR spectra was processed using NMRPipe software (http://spin.niddk.nih.gov/NMRPipe/). ${ }^{23,24}$ Peak assignments were made using the SpinAssign program on the PRIMe website (http://prime.psc.riken.jp/). ${ }^{25-27}$

To evaluate the water-insoluble seaweed components, lyophilized samples were powdered and processed by various procedures to identify the best extraction method (Supplementary Table 2), and the optimal-modified process was used for all further analyses. In the optimal process, the lyophilized samples were roughly crushed in a food blender and then powdered using an Automill machine. The powdered samples were pretreated twice with hexafluoroacetone (HFA) to eliminate some of the overlapping protein signals and evaporated. To remove any small molecules, the HFA-pretreated samples were cycled thrice through a $5 \mathrm{~min}$ incubation with ultrapure water at $50{ }^{\circ} \mathrm{C}$, followed by supernatant removal before incubating three more times in methanol at $50{ }^{\circ} \mathrm{C}$ for $5 \mathrm{~min}$ with subsequent supernatant removal. At this point, the samples were milled 36 times in a ball mill machine (Fritsch Japan Co., Ltd., Kanagawa, Japan) for 10 min each with 10 min intervals to effectively dissolve the cell wall components in the DMSO- $\mathrm{d}_{6} /$ Pyridine- $_{5}$ solvent $(4 / 1$,

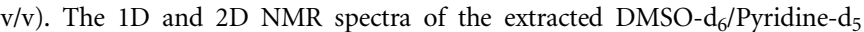
samples were acquired at $298 \mathrm{~K}$ using a $700 \mathrm{MHz}$ Bruker Biospin instrument equipped as described above (Bruker Biospin); the central DMSO solvent peak was used as the internal reference $\left({ }^{13} \mathrm{C} 40.03,{ }^{1} \mathrm{H} 2.582\right.$ p.p.m.). For the ${ }^{1} \mathrm{H}-{ }^{13} \mathrm{C}$ HSQC NMR measurements, a total of 256 complex f1 $\left({ }^{13} \mathrm{C}\right)$ and 1024 complex $\mathrm{f} 2\left({ }^{1} \mathrm{H}\right)$ points were recorded using 32 scans per $\mathrm{f} 1$ increment. The obtained spectral widths were 150 and 14 p.p.m. for $\mathrm{f} 1$ and f2, respectively. Alginic acid, fucoidan, galactan, laminaran (Sigma-Aldrich Japan Co., Ltd., Tokyo, Japan) and agar (Wako Pure Chemical Industries, Ltd., Osaka, Japan) were analyzed as standard compounds for assigning the detected signals.

\section{ATR-FTIR spectroscopy}

The ATR-FTIR spectra $\left(400-4000 \mathrm{~cm}^{-1}\right)$ of seaweed samples lyophilized and powdered as described above were obtained using a Nicolet 6700 FT-IR spectrometer (Thermo Fisher Scientific Inc., Waltham, MA, USA) with $\mathrm{KBr}$ disks. A smart iTR accessory with a high-pressure clamp (Thermo Fisher Scientific Inc.) was used for the ATR-FTIR measurements and collected 10 scans each of the background and sample spectra. Both baseline and ATR corrections for the penetration depth and frequency variations were applied using the OMNIC software (Thermo Fisher Scientific Inc., Waltham, MA, USA) supplied with the equipment.

\section{Multivariate statistical analysis}

The ${ }^{1} \mathrm{H}-\mathrm{NMR}$ data were reduced by subdividing the spectra into sequential designated 0.04 p.p.m. regions between the ${ }^{1} \mathrm{H}$ chemical shifts of 0.0-9.0. Each region was integrated and normalized to the DSS integral regions after excluding any water resonances. The ATR-FTIR spectral data were integrated to 1869 data points throughout the $400-4000 \mathrm{~cm}^{-1}$ spectral range using the OMNIC software supplied with the equipment. The ${ }^{1} \mathrm{H}-\mathrm{NMR}$ and ATR-FTIR spectral data were statistically evaluated using both principal component analysis (PCA) and hierarchical cluster analysis (HCA) on the ' $\mathrm{R}$ ' software (http://www.R-project.org/). Both PCA and HCA were used to explore any biomass component clustering based on intrinsic biochemical similarities. PCA and HCA facilitate the simultaneous comparison of a large number of complex objects, such as those in NMR and ATR-FTIR spectra, and can be used to statistically summarize the signature of intersample variations and relationships within the multivariate data. ${ }^{6,8,10-13,18,19}$ In PCA, the PCs are calculated so that each component is a linear combination of the original variables orthogonal to the other PCs and with each successive component describing progressively less data variation. The data were visualized in the form of PC score and loading plots. The PC score plots can yield the sample distribution based on the biochemical variation in the data set and highlight groupings within a data set. Each coordinate in a score plot represents an individual sample, and each coordinate in a loading plot represents one NMR or ATRFTIR spectral data point related to the biomass components. Therefore, a loading plot provides information on the spectral position responsible for the coordinated sample clusters in the corresponding score plot. In HCA, the data were represented using a hierarchical or tree-like structure calculated via the agglomerative method. The resulting hierarchical dendrogram can provide information regarding the relationships between the biomass samples by grouping them on the basis of their similarities, which allows for a visual understanding of both the fine and coarse inclusive relations between clusters.

\section{RESULTS AND DISCUSSION}

Evaluation of water-soluble components in seaweed biomass by NMR spectroscopy

To evaluate the water-soluble components in seaweed biomass, samples dissolved in $\mathrm{D}_{2} \mathrm{O}$ were analyzed by NMR using the WG and CPMG pulse sequence programs (Supplementary Figure 1). A comparison between the CPMG and WG spectra of red algae is likely to reveal a signal enhancement derived from the monomeric or 
oligomeric sugar components and amino acids in the spectra (Supplementary Figures 1i-k). In contrast, a comparison between the brown algae spectra is likely to highlight the signals at 3.87, 3.79, 3.75 and 3.67 p.p.m., possibly derived from mannitol (Supplementary Figures 1l-t). All spectral data were then processed by PCA to determine the individual characteristics of the samples because PCA can facilitate the simultaneous comparison of a large amount of NMR spectral data and can statistically identify both similarities and dissimilarities in the intersample variations within multivariate data. Through the PCA score plots of the ${ }^{1} \mathrm{H}-\mathrm{NMR}$ data matrices, the water-soluble component profiles of these seaweeds tended to cluster according to their taxonomical grouping (that is, green, red and brown algae; Figure 1a). Thus, each NMR spectrum of water-soluble components from the same taxonomical group had a relatively high similarity to other samples of that group. In other words, PCA was capable of revealing the type and proportions of the chemical constituents within the taxonomical groupings. To identify the largest differences in the NMR chemical shifts and chemical components that contribute to the intergroup variations, loading plots were analyzed to provide information on the spectral positions responsible for the clustered positions in the corresponding sample score plots. Some water-soluble components, such as mannitol, D-galactose and
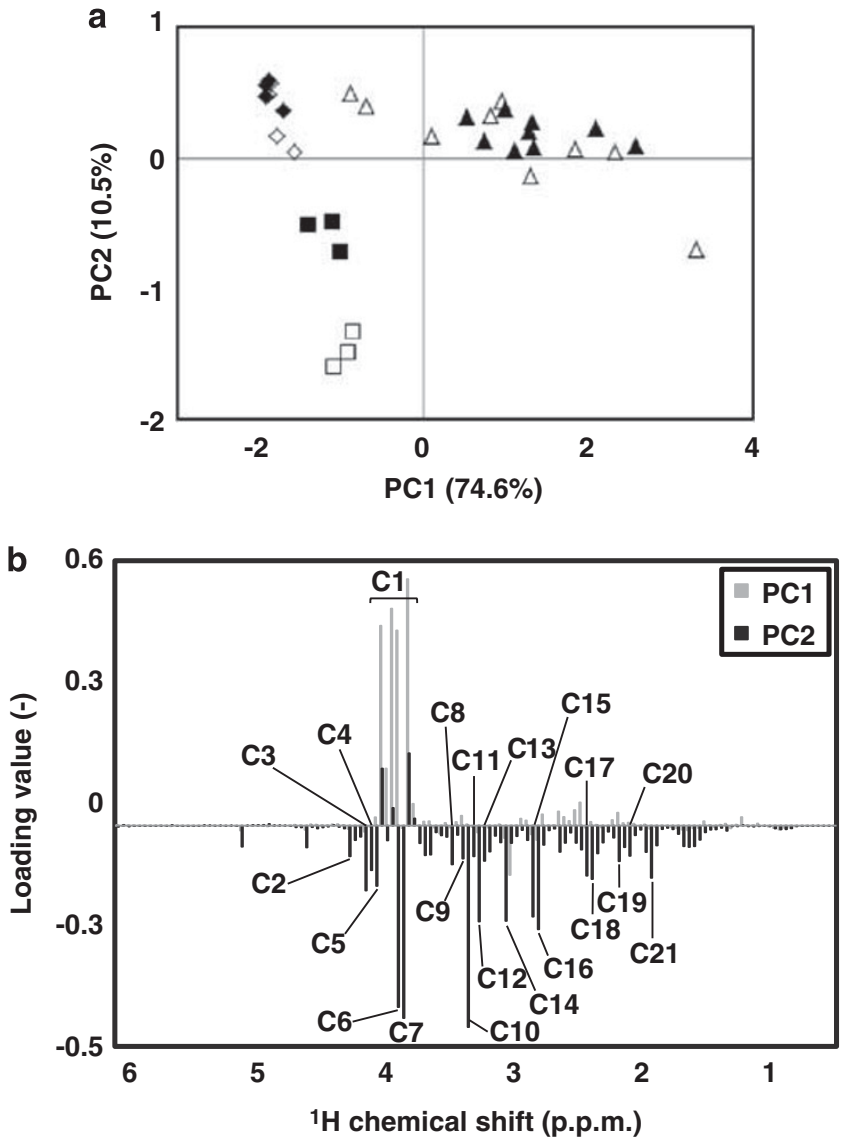

Figure 1 Profiles of the water-soluble seaweed components evaluated by PCA based on their ${ }^{1} \mathrm{H}-\mathrm{NMR}$ spectra. (a) PCA score plot computed from green algae CPMG $(\diamond)$, green algae WG $(\diamond)$, red algae CPMG $(\boldsymbol{\square})$, red algae WG $(\square)$, brown algae CPMG $(\boldsymbol{\Delta})$ and brown algae WG $(\triangle)$ spectra; (b) PCA loading plot that provided information regarding the spectral regions responsible for the coordinate and cluster positions for the samples in the corresponding score plots; numbers (1-21) contributed chemical compounds determined by the SpinAssign program and listed in Table 1.
L-glutamate, contributed to the clustering and were labeled by the SpinAssign program according to the loading plots data (Figures $1 \mathrm{~b}$ and 2a and Table 1). Mannitol was characteristic of brown algae, whereas certain sugars, such as D-galactose and D-fructose, and amino acids, such as L-glutamate and L-aspartate, were characteristic of red algae. As mannitol is known to be abundant in brown algae ${ }^{1}$ and D-galactose is an abundant constituent sugar in carrageenan and agar from red algae, ${ }^{1}$ the biomass profiling of the water-soluble components was able to identify individual characteristics among the various seaweeds. Surprisingly, the PCA results were indicative of clustering according to the differences between the taxonomic groups, whereas the general data seemed to define these separations according to the differences in pulse sequence data in the WG and CPMG spectra. Therefore, these results revealed that the NMR profiles of the water-soluble seaweed components possessed great diversity based on their taxonomic grouping and were unaffected by differences in the pulse sequence data.

\section{Evaluation of water-insoluble components in seaweed biomass by NMR spectroscopy}

The method for extracting water-insoluble components from seaweed biomass was first developed by examining, modifying and optimizing a physical pretreatment and extraction process using Eisenia bicyclis (Supplementary Table 2). Although the number and intensity of the NMR signals for the test material pretreated using a ball mill machine was better than that for other pretreatment systems, such as using a food blender and Automill machine, the target biomass signals overlapped with the protein signals (Supplementary Figure 2). Therefore, the extraction process was further modified by removing the small molecules via water and methanol extractions and eliminating the protein signals via HFA extractions as described above (Supplementary Figure 3) before being deemed the optimal extraction process for the water-insoluble biomass components (Supplementary Figure 4).

Next, the biomass profiles of the extracted water-insoluble components prepared as described above and extracted with a DMSO- $\mathrm{d}_{6} /$ Pyridine- $\mathrm{d}_{5}$ solvent $(4 / 1, \mathrm{v} / \mathrm{v})$ were evaluated using PCA score plots (Supplementary Figure 5). The water-insoluble component profiles for these various seaweeds tended to cluster according to their taxonomical differences, which indicated that the NMR spectra of components in the same taxonomic groups were similar relative to those from other taxonomic groups. Moreover, to obtain more detailed information regarding the chemical components in seaweed biomass, both the water-soluble and water-insoluble component profiles were evaluated using 2D HSQC NMR spectra (Figure 2 and Supplementary Tables 3 and 4). Standards of the detected compounds, such as laminaran and agar, were measured to assign the peaks detected in the water-insoluble HSQC NMR spectra. The distribution patterns of the detected peaks in the HSQC NMR spectra were characteristically different in regard to their taxonomical grouping (Figure 2), which was consistent with the PCA results. Moreover, the peaks detected in HSQC NMR spectra by the NMR-based metabolomics approach can be annotated as candidate chemical compounds; therefore, the detected peaks were further analyzed to determine any candidate compounds and are listed in Supplementary Tables 3 and 4. The NMR signals derived from laminaran (a major brown algae storage glucan) were detected as characteristic components for brown algae, whereas the signals derived from agar (a gelatinous substance derived from a polysaccharide that accumulates in the agarophyte red algae cell walls) were detected as characteristic components for red algae. ${ }^{1}$ Moreover, some signals derived from 
Table 1 List of the chemical compounds attributed to the separation based on the PCA and determined by the SpinAssign program

\begin{tabular}{|c|c|c|c|c|c|c|}
\hline \multirow{2}{*}{$\begin{array}{l}\text { Annotation number } \\
\text { C1 }\end{array}$} & \multirow{2}{*}{$\begin{array}{c}{ }^{1} \mathrm{H} \text { chemical shift (p.p.m.) } \\
3.87\end{array}$} & \multicolumn{5}{|c|}{ Candidate metabolites } \\
\hline & & Mannitol & & & & \\
\hline $\mathrm{C} 1$ & 3.79 & Mannitol & & & & \\
\hline $\mathrm{C} 1$ & 3.75 & Mannitol & & & & \\
\hline $\mathrm{C} 1$ & 3.67 & Mannitol & & & & \\
\hline $\mathrm{C} 2$ & 4.11 & L-Proline & D-Fructose & & & \\
\hline $\mathrm{C} 3$ & 3.99 & L-Phenylalanine & Galactitol & D-Galactose & D-Fructose & \\
\hline C4 & 3.95 & Gentiobiose & D-Galactose & & & \\
\hline C5 & 3.91 & L-Aspartate & Gentiobiose & D-Glucose & D-Galactose & D-Fructose \\
\hline C6 & 3.75 & L-Iditol & L-Homoserine & L-Glutamine & L-Glutamate & L-Arginine \\
\hline $\mathrm{C7}$ & 3.71 & L-Iditol & Gentiobiose & Galactitol & D-Glucose & D-Galactose \\
\hline $\mathrm{C8}$ & 3.36 & Gentiobiose & & & & \\
\hline C9 & 3.28 & L-Phenylalanine & Gentiobiose & & & \\
\hline C10 & 3.24 & L-Phenylalanine & L-Arginine & Gentiobiose & D-Glucose & \\
\hline C11 & 3.2 & L-Tyrosine & & & & \\
\hline C12 & 3.16 & - & & & & \\
\hline C13 & 3.12 & L-Phenylalanine & & & & \\
\hline C14 & 2.96 & L-Asparagine & & & & \\
\hline C15 & 2.76 & L-Aspartate & & & & \\
\hline C16 & 2.72 & - & & & & \\
\hline C17 & 2.36 & L-Proline & L-Glutamate & & & \\
\hline C18 & 2.32 & L-Proline & L-Glutamate & & & \\
\hline C19 & 2.12 & L-Homoserine & L-Glutamine & L-Glutamate & & \\
\hline C2O & 2.04 & L-Proline & L-Homoserine & L-Glutamate & & \\
\hline $\mathrm{C} 21$ & 1.88 & L-Arginine & Acetate & & & \\
\hline
\end{tabular}

polysaccharides corresponding to the cell wall components in land plants, namely, xylopyranose and glucopyranose, were detected as characteristic components for green algae, which suggested that green algae had plant cell wall-like structures in their water-insoluble components. Taken together, this strategy for profiling both the water-soluble and water-insoluble components of seaweed biomass was capable of evaluating individual characteristics among various seaweeds.

Evaluation of solid-state constituents in seaweed biomass by ATRFTIR spectroscopy

ATR-FTIR measurements were performed on freeze-dried and powdered seaweed samples to evaluate their solid-state biomass constituents, and the resulting PCA score plots of the data matrices showed that the solid-state constituent profiles of these seaweeds were also likely to cluster according to their taxonomical grouping (Supplementary Figure 6). Therefore, this approach to profiling the constituents of solid and water-soluble and water-insoluble seaweed biomass was capable of identifying individual characteristics among various seaweeds.

Chemical profiling of water-soluble, water-insoluble and solid-state biomass from various seaweeds

The water-soluble, water-insoluble and solid-state biomass profiles of these seaweeds were compared via reanalysis using the HCA of the obtained NMR and ATR-FTIR spectral data (Figure 3 and Supplementary Figure 7). An HCA dendrogram allows for the visualization and understanding of both the fine and coarse inclusive relationships between clusters. For example, the HCA dendrogram of water-soluble biomass profiles was roughly clustered into four groups (Figure 3a) with two groups, which consisted of brown algae, clustered to the left and the other two groups clustered, which consisting of green and red algae, to the right. In this way, the HCA dendrograms of the profile components distinctively visualized their intergroup relations. Each type of profile clustered according to the differences between their green, red and brown algae characteristics. In particular, the profiles of the brown algae biomass were more uniquely characteristic than those of the green and red algae. Furthermore, the seaweed biomass profiles were largely different from those of land plants. ${ }^{14,15}$ Thus, the chemical components of seaweed observed here might reflect the evolution of plants because the evolutionary distance of brown algae from land plants is greater than that of the other algae. ${ }^{28,29}$ Taken together, these profiling strategies for describing the water-soluble, water-insoluble and solidstate components of seaweed were capable of evaluating the individual characteristics of different types of seaweed. This comprehensive approach will provide a strategy and technological platform for evaluating the compositional variations and diversity of complicated macromolecular mixtures of various seaweeds, and opens new avenues for understanding biomacromolecules in natural ecosystems.

The biomass profiling of seaweeds has been largely unexplored in terms of the comprehensive characterization afforded by the NMRbased metabolomics approach. Therefore, this study was the first attempt to understand seaweed biomass profiles via comprehensive metabolomics characterizations with multivariate statistical analyses. The water-soluble, water-insoluble and solid-state components of seaweed biomass were selected to develop these strategies as an initial step in the chemical profiling of complex biochemical mixtures. The characterization of the water-soluble components via an NMR-based metabolomics approach has been used extensively to study metabolites in a wide range of biological systems in various environments, ${ }^{6-13}$ and is capable of creating assignments using our database (that is, SpinAssign program at the PRIMe website)..$^{25}$ The water-insoluble 

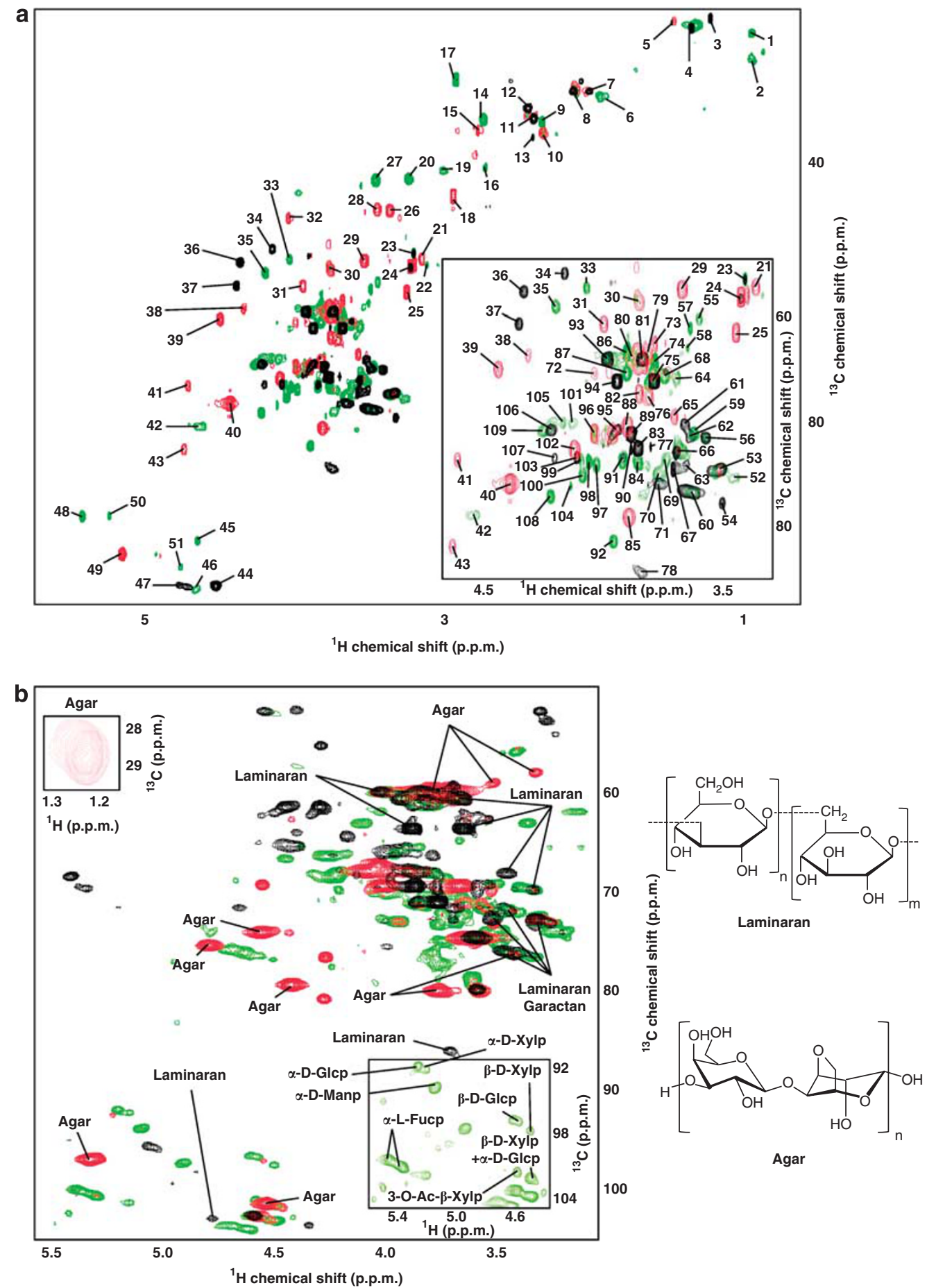

Figure $2{ }^{1} \mathrm{H}-{ }^{13} \mathrm{C}$ HSQC spectra of both the water-soluble (a) and water-insoluble (b) component profiles for a variety of seaweeds. Superimposed ${ }^{1} \mathrm{H}-{ }^{13} \mathrm{C}$ HSQC spectra of green (green), red (red) and brown algae (black); numbers (1-109) represent the determined chemical shifts assigned by the SpinAssign program and are listed in Supplementary Table 3.

components (including the cell wall components and polysaccharide storage) were important constituents in seaweeds; therefore, an extraction method before the NMR measurements was developed via the modification and optimization of physical pretreatments and extraction processes. As both the water-soluble and water-insoluble components were extractable, the solid-state constituents were also analyzed to obtain an intact biomass profile.
In this study, the ATR-FTIR measurements were used to evaluate the chemical profile of the solid-state biomass constituents. Such measurements enable samples to be examined directly in the solid state without further preparation; therefore, ATR-FTIR has the advantages of only requiring a small amount of the sample; rapid measurement times, which allow for a high throughput; and both easy and simple operation. High-throughput ATR-FTIR 

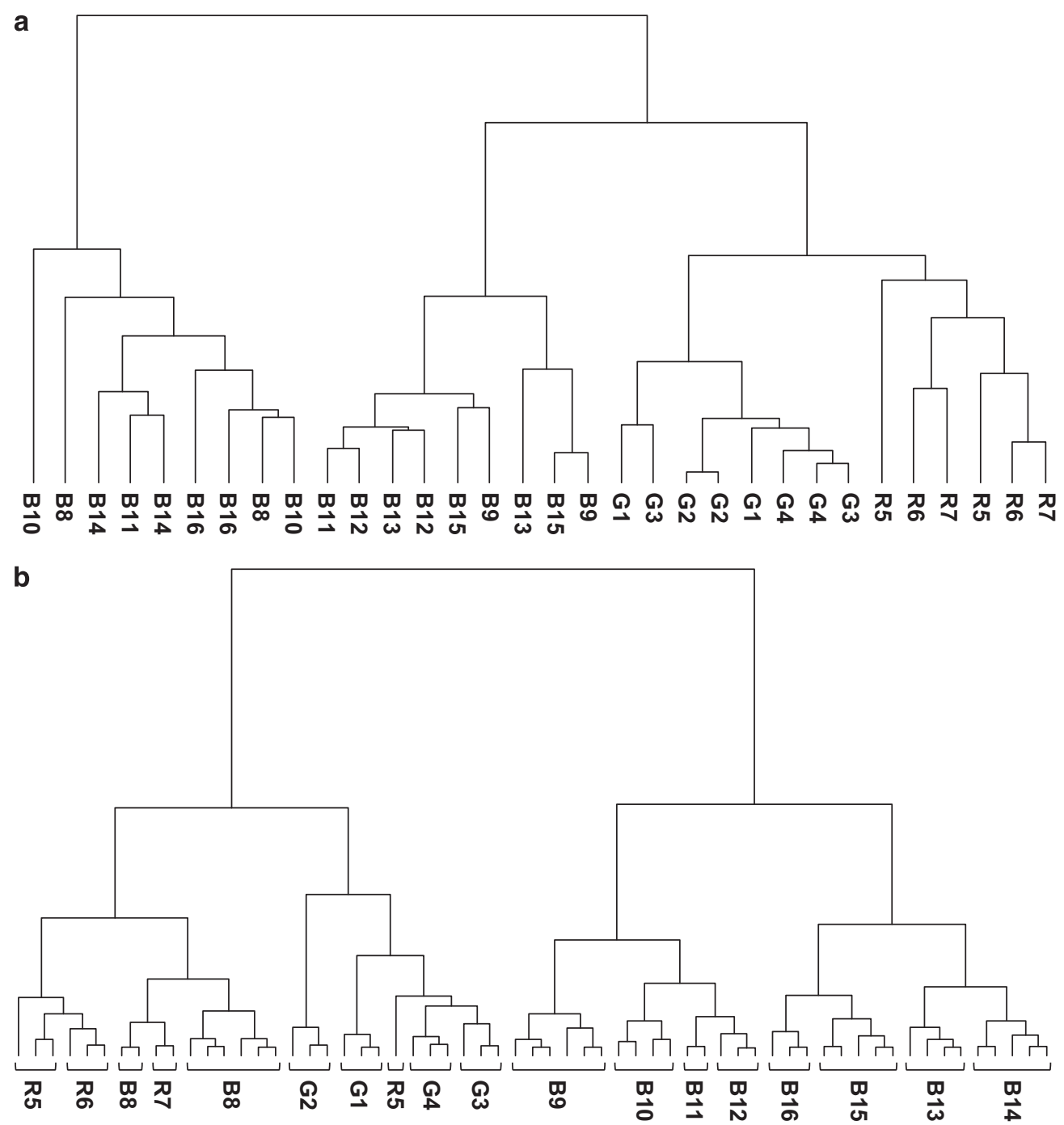

Figure 3 Water-soluble (a) and solid-state (b) biomass profiles for the seaweeds evaluated by HCA based on the ${ }^{1} \mathrm{H}-\mathrm{NMR}$ and ATR-FTIR spectra, respectively.

measurements combine with multivariate statistical analysis to become a powerful tool for evaluating and identifying individual characteristics of solid-state biomass constituents on the basis of their chemical profile patterns. However, the disadvantage of this approach is the difficulty of linking the representative signals or peaks to the individual characteristics and variations in any chemical compounds and structures. Therefore, further analysis, such as solid-state NMR measurements, would be useful to specify certain factors that contribute to these intersample variations and relationships by identifying and assigning the chemical and/or structural characteristics of the solid-state biomass constituents. Actually, the description of a chemical profiling strategy in this report is the first step in developing an analytical method and comprehensive approach for the characterization, evaluation and elucidation of the chemical and structural interactions and relationships between complex biomacromolecular mixtures in natural biomass. In the next step, the use of solid-state NMR measurements to evaluate the solid-state constituents will be considered and combined with existing methods to assess physicochemical properties, such as thermodynamic characterization, for the development of new methodologies in future works.
This study focused on the development of profiling techniques for evaluating the water-soluble, water-insoluble and solid-state components of seaweed biomass to address the challenges in determining, comparing and understanding their biochemical complexities. NMR measurements of the water-insoluble seaweed components were accomplished via the modification and optimization of a physical pretreatment and extraction process based on a DMSO- $\mathrm{d}_{6} /$ Pyridine$\mathrm{d}_{5}(4 / 1, \mathrm{v} / \mathrm{v})$ solvent system. Evaluating all of the compositional profiles obtained via NMR and ATR-FTIR spectroscopy using multivariate statistical analysis, such as PCA and HCA, provided a procedural plan capable of distinguishing the individual characteristics of various seaweeds. The development of new methodologies incorporating this approach will help clarify the relationship between complex biomacromolecules in natural products, elucidate their supramolecular structures and functions in natural ecosystems, and provide a promising new field in polymer science.

\section{ACKNOWLEDGEMENTS}

We thank Eisuke Chikayama, Yuuri Tsuboi and Amiu Shino (RIKEN) for their stimulating discussion and technical advice regarding NMR measurements and analyses. We also thank Jiro Tanaka (Tokyo University of Marine Science and 
Technology), Osamu Takahashi and Yuji Omori (Yokosuka City Museum) for technical assistance in collecting and identifying the seaweeds. This research was supported in part by Grants-in-Aid for Scientific Research for challenging exploratory research and the Ministry of Education, Culture, Sports, Science and Technology, Japan (MEXT to JK). The work was also supported in part by grants from the New Energy and Industrial Technology Development Organization (NEDO to JK), and the Adaptable and Seamless Technology Transfer Program through Target-driven R\&D, Japan Science and Technology Agency (JST to JK).

1 Percival, E. The polysaccharides of green, red and brown seaweeds: their basic structure, biosynthesis and function. Brit. Phycol. J. 14, 103-117 (1979).

2 Samir, M. A. S. A., Alloin, F. \& Dufresne, A. Review of recent research into cellulosic whiskers, their properties and their application in nanocomposite field. Biomacromolecules 6, 612-626 (2005).

3 Ministry of Agriculture, Forestry and Fisheries in Japan. White Paper on Fisheries H22, 10-41 (2010).

4 Nisizawa, K., Noda, H., Kikuchi, R. \& Watanabe, T. The main seaweed foods in Japan. Hydrobiologia 151/152, 5-29 (1987).

5 Clément, M. J., Tissot, B., Chevolot, L., Adjadj, E., Du, Y., Curmi, P. A. \& Daniel, R. NMR characterization and molecular modeling of fucoidan showing the importance of oligosaccharide branching in its anticomplementary activity. Glycobiology 20, 883894 (2010).

6 Bundy, J. G., Davey, M. P. \& Viant, M. R. Environmental metabolomics: a critical review and future perspectives. Metabolomics 5, 3-21 (2009).

7 Date, Y., Nakanishi, Y., Fukuda, S., Kato, T., Tsuneda, S., Ohno, H. \& Kikuchi, J. New monitoring approach for metabolic dynamics in microbial ecosystems using stableisotope-labeling technologies. J. Biosci. Bioeng. 110, 87-93 (2010)

8 Everroad, R. C., Yoshida, S., Tsuboi, Y., Date, Y., Kikuchi, J. \& Moriya, S. Concentration of metabolites from low-density planktonic communities for environmental metabolomics using nuclear magnetic resonance spectroscopy. J. Vis. Exp. 62, e3163 (2012).

9 Frimmersdorf, E., Horatzek, S., Pelnikevich, A., Wiehlmann, L. \& Schomburg, D. How Pseudomonas aeruginosa adapts to various environments: a metabolomic approach. Environ. Microbiol. 12, 1734-1747 (2010).

10 Fukuda, S., Nakanishi, Y., Chikayama, E., Ohno, H., Hino, T. \& Kikuchi, J. Evaluation and characterization of bacterial metabolic dynamics with a novel profiling technique, real-time metabolotyping. PLOS ONE 4, e4893 (2009).

11 Hughes, S. L., Bundy, J. G., Want, E. J., Kille, P. \& Sturzenbaum, S. R. The metabolomic responses of Caenorhabditis elegans to cadmium are largely independent of metallothionein status, but dominated by changes in cystathionine and phytochelatins. J. Proteome Res. 8, 3512-3519 (2009).

12 Nakanishi, Y., Fukuda, S., Chikayama, E., Kimura, Y., Ohno, H. \& Kikuchi, J. Dynamic omics approach identifies nutrition-mediated microbial interactions. J. Proteome Res. 10, 824-836 (2011)

13 Sekiyama, Y., Chikayama, E. \& Kikuchi, J. Evaluation of a semipolar solvent system as a step toward heteronuclear multidimensional NMR-based metabolomics for ${ }^{13}$ C-labeled bacteria, plants, and animals. Anal. Chem 83, 719-726 (2011).

14 Hedenström, M., Lindström, S. W., Öman, T., Lu, F., Gerber, L., Schatz, P., Sundberg, B. \& Ralph, J. Identification of lignin and polysaccharide modifications in populus wood by chemometric analysis of 2D NMR spectra from dissolved cell walls. Mol. Plant 2, 933-942 (2009)

$15 \mathrm{Kim}, \mathrm{H}$. \& Ralph, J. Solution-state 2D NMR of ball-milled plant cell wall gels in DMSO-d $\mathrm{d}_{6} /$ Pyridine- $\mathrm{d}_{5}$. Org. Biomol. Chem. 8, 576-591 (2010).

16 Piotto, M., Saudek, V. \& Skelener, V. Gradient-tailored excitation for single-quantum NMR spectroscopy of aqueous solutions. J. Biomol. NMR 2, 661-665 (1992).

17 Claridge, T. D. W. High-Resolution NMR Techniques in Organic Chemistry (Elsevier Sciences Ltd, Pergamon 19, 1999).

18 Solanky, K. S., Bailey, N. J. C., Beckwith-Hall, B. M., Davis, A., Bingham, S., Holmes, E., Nicholson, J. K. \& Cassidy, A. Application of biofluid ${ }^{1} \mathrm{H}$ nuclear magnetic resonance-based metabonomic techniques for the analysis of the biochemical effects of dietary isoflavones on human plasma profile. Anal. Biochem. 323, 197-204 (2003).

19 Tiziani, S., Kang, Y., Choi, J. S., Roberts, W. \& Paternostro, G. Metabolomic high-content nuclear magnetic resonance-based drug screening of a kinase inhibitor library. Nat. Commun. 2, 545 (2011).

20 Kikuchi, J. \& Hirayama, T. Practical aspects of uniform stable isotope labeling of higher plants for heteronuclear NMR-based metabolomics. Methods Mol. Biol. 358, 273-286 (2007).

21 Sekiyama, Y., Chikayama, E. \& Kikuchi, J. Profiling polar and semipolar plant metabolites throughout extraction processes using a combined solution-state and high-resolution magic angle spinning NMR approach. Anal. Chem. 82, 1643-1652 (2010).

22 Sekiyama, Y. \& Kikuchi, J. Towards dynamic metabolic network measurements by multi-dimensional NMR-based fluxomics. Phytochemistry 68, 2320-2329 (2007).

23 Delaglio, F., Grzesiek, S., Vuister, G. W., Zhu, G., Pfeifer, J. \& Bax, A. NMRPipe: a multidimensional spectral processing system based on UNIX pipes. J. Biomol. NMR 6, 277-293 (1995).

24 Kikuchi, J., Shinozaki, K. \& Hirayama, T. Stable isotope labeling of Arabidopsis thaliana for an NMR-based metabolomics approach. Plant Cell Physiol. 45, 1099-1104 (2004).

25 Akiyama, K., Chikayama, E., Yuasa, H., Shimada, Y., Tohge, T., Shinozaki, K., Hirai, M Y., Sakurai, T., Kikuchi, J. \& Saito, K. PRIMe: a Web site that assembles tools for metabolomics and transcriptomics. In Silico Biol. 8, 339-345 (2008).

26 Chikayama, E., Sekiyama, Y., Okamoto, M., Nakanishi, Y., Tsuboi, Y., Akiyama, K., Saito, K., Shinozaki, K. \& Kikuchi, J. Statistical indices for simultaneous large-scale metabolite detections for a single NMR spectrum. Anal. Chem. 82, 1653-1658 (2010).

27 Chikayama, E., Suto, M., Nishihara, T., Shinozaki, K., Hirayama, T. \& Kikuchi, J. Systematic NMR analysis of stable isotope labeled metabolite mixtures in plant and animal systems: coarse grained views of metabolic pathways. PLoS ONE 3, e3805 (2008).

28 Martone, P. T., Estevez, J. M., Lu, F., Ruel, K., Denny, M. W., Somerville, C. \& Ralph, J. Discovery of lignin in seaweed reveals convergent evolution of cell-wall architecture. Curr. Biol. 19, 169-175 (2009)

29 Yoon, H. S., Hackett, J. D., Ciniglia, C., Pinto, G. \& Bhattacharya, D. A molecular timeline for the origin of photosynthetic eukaryotes. Mol. Biol. Evol. 21, 809-818 (2004)

This work is licensed under the Creative Commons Attribution-NonCommercial-No Derivative Works 3.0 Unported License. To view a copy of this license, visit http:// creativecommons.org/licenses/by-nc-nd/3.0/

Supplementary Information accompanies the paper on Polymer Journal website (http://www.nature.com/pj) 